im Bereich von Sport- und Großveranstaltungen und gegenüber vermeintlich unpolitischen Akteur_innen aufgrund gemachter Erfahrungen im Umgang mit ,den Linken“ verändert hat, hätte sich als mögliche Erweiterung angeboten.

\title{
Kötzing, Andreas (Hrsg.): Bilder der Allmacht. Die Staatssicherheit in Film und Fernsehen, 336 S., Wallstein, Göttingen 2018.
}

\author{
Jörg Requate \\ Online publiziert: 13. Juli 2020 \\ (C) Der/die Autor(en) 2020
}

Die DDR war nicht gerade ein Staat, der geeignet gewesen wäre, den Glamour Hollywoods anzuziehen. Der Stasi gelang in gewisser Weise allerdings auch dies: Der Mauerbau und die besondere Lage Berlins beflügelten schnell die Fantasien von Filmschaffenden und so entstanden gerade in den 1960er Jahren eine Reihe hochkarätig besetzter Filme, in denen sich etwa Paul Newman (,Der zerrissene Vorhang“, 1966) oder Richard Burton (,Der Spion, der aus der Kälte kam“, 1965) mit den Machenschaften der Stasi auseinandersetzen mussten. Mit „Das Leben der Anderen“ gelang Florian Henckel von Donnersmarck im Jahr 2006 schließlich eine oscarprämierte Darstellung der Stasi, was ihr noch einmal die ganz große internationale Bühne verschaffte. Der Begriff ,Stasi` sei damit gleichsam zu einem globalen Synonym für Geheimdienste geworden, schrieb Timothy Garton Ash in einem Beitrag über die Wirkung und Bedeutung des Films, mit dem sich auch Tobias EbbrechtHartmann in dem vorliegenden Band auseinandersetzt.

Wie der von Andreas Kötzing herausgegebene Band über „Die Staatssicherheit in Film und Fernsehen“" zeigt, bilden diese Beispiele jedoch nur einen winzigen Ausschnitt innerhalb der gesamten filmischen Auseinandersetzung mit der Stasi. Den Ausgangspunkt des Buches liefert die Beobachtung, dass die Stasi in der medialen Auseinandersetzung eine ungebrochen hohe Aufmerksamkeit genießt, und der Herausgeber geht davon aus, dass dies aller Voraussicht nach auch in absehbarer Zukunft noch so bleiben wird. Der Band beschränkt sich jedoch nicht nur auf die Zeit nach

J. Requate $(\square)$

Universität Kassel, Kassel, Deutschland

E-Mail: joerg.requate@uni-kassel.de 
dem Ende der DDR, sondern spannt einen weiten Bogen von den frühen 1950er Jahren bis in die jüngste Vergangenheit. Sinnvollerweise wird mit dem Jahr 1989/90 ein Schnitt gesetzt, da sich mit der Auflösung der DDR die Parameter für die Darstellung deutlich veränderten. War die filmische Darstellung des DDR-Geheimdienstes bis zu diesem Zeitpunkt Teil der propagandistischen Auseinandersetzung zwischen den ,Blöcken“, wurde das Agieren der Stasi nach 1989/90 zu einem Gegenstand, der sich ,Zwischen politischer Aufarbeitung und medialer Unterhaltung“ bewegte.

Den Schwerpunkt für die Zeit bis 1989/90 bildet die Auseinandersetzung mit den filmischen Produktionen, die innerhalb der DDR entstanden sind und damit im Wesentlichen Teil der Selbstdarstellung der Staatssicherheit waren. Dabei ist durchaus überraschend, wie früh und wie offensiv die Stasi darum bemüht war, eine gewisse öffentliche Präsenz zu zeigen. Den Ausgangspunkt bildete, wie der Beitrag von Daniela Münkel und Elke Stadelmann-Wenz zeigt, der Aufstand vom 17. Juni 1953. Hatte das Ministerium für Staatssicherheit (MfS) bis zu diesem Zeitpunkt weitgehend unbeachtet von der Öffentlichkeit agiert, wechselten die maßgeblichen Akteure nun ihre Strategie und starteten eine regelrechte ,Image- und Öffentlichkeitskampagne“. Insbesondere wurde die im Rahmen der „Aktion ,Feuerwerk“" vorgenommene Verhaftung von 218 Personen, die unter dem Verdacht standen, für die Bundesrepublik spioniert zu haben, propagandistisch ausgeschlachtet. Die breit angelegte Medienkampagne - filmische Darstellungen waren hier integriert, bildeten aber nicht das Zentrum - sollte deutlich machen, dass der junge Staat bereit und in der Lage war, sich gegen seine Feinde zur Wehr zu setzen. Die Präsentation der eigenen Macht setzte somit früh den entscheidenden Akzent für die weiteren medialen Darstellungen der Stasi. Dies zeigte sich nicht nur in dokumentarischpropagandistischen Beiträgen, sondern auch in einer Reihe von Spielfilmen, wie vor allem Kötzing in seiner Untersuchung von DEFA-Spielfilmen zu dem Thema hervorhebt. Jenseits der medialen Inszenierung von Macht versuchten sowohl dokumentarische als auch insbesondere fiktionale Darstellungen darüber hinaus, die Stasi in ihrer vermeintlich beschützenden Funktion darzustellen. Besonders geeignet hierzu war ,der Spion', der - wie Michael Grisko in seinem Beitrag über die Fernsehserie „Das unsichtbare Visier“ zeigt - zu einer sozialistischen Heldenfigur aufgebaut wurde. Gerade auf fiktionaler Ebene erwiesen sich hier die Darstellungen aus der DDR in gewisser Weise als komplementär zu den westlichen Spionagefilmen des Kalten Krieges. Christoph Classen zeigt jedoch, dass die Darstellung der östlichen Spione in den Spielfilmen des Westens im Einzelnen sehr viel differenzierter ausfiel, als dies auf den ersten Blick erscheinen mag. Die Trennlinie zwischen moralisch anständigem und verwerflichem Handeln verlief, so Classen, zunehmend eher innerhalb als zwischen den Lagern. Classen betont allerdings auch, dass die Filme sich nicht wirklich für die Stasi oder den KGB interessierten. Ihr eigentliches Thema sei „die Bigotterie und die Unglaubwürdigkeit des Westens“ (S. 93) gewesen. Unverkennbar ist allerdings, dass sich hier ein Genre entwickelte, das nicht zuletzt auch der Unterhaltung diente und entsprechende Publikumserwartungen zu erfüllen hatte, um zu funktionieren.

Insofern entsteht - vermutlich unbeabsichtigt - ein etwas schiefer Eindruck, wenn der Aspekt der „,medialen Unterhaltung“ nur in die Überschrift des zweiten Teils des Buches aufgenommen wird. Der Beitrag von Anita Krätzner-Ebert zur Darstel- 
lung der Stasi in der Fernsehreihe „Tatort“ durchbricht zudem die klare chronologische Teilung des Buches, sodass auch hier schon klar wird, in welchem Maße der Unterhaltungsaspekt in den fiktionalen Darstellungen durchgehend zentral ist. Die ,Allmacht", oder besser die große und schwer durchschaubare Macht der Stasi bildet insofern wohl tatsächlich denjenigen Aspekt, der alle filmischen Auseinandersetzungen mit dem Thema verbindet. Die Beiträge zeigen aber auch, dass in der Zusammenschau ein durchaus vielfältiges Bild entsteht. Zwar hat Kötzing wohl recht mit seiner Feststellung, dass sich die Differenzierungen, die sich in der wissenschaftlichen Literatur zur Stasi inzwischen zeigen, ,nur bedingt in filmischen Inszenierungen des MfS widerspiegeln“ (S. 38). Allerdings wäre zu fragen, ob hier nicht letztlich doch stärker zwischen dokumentarischer und fiktionaler Auseinandersetzung mit dem Thema zu unterscheiden wäre. Filme wie „Das Leben der Anderen“ oder „Gundermann“, der mehr oder weniger zeitgleich mit dem Erscheinen des Bandes 2018 in die Kinos gekommen ist und insofern noch nicht wirklich berücksichtigt werden konnte, liefern ganz unterschiedliche künstlerische Annäherungen an das Thema mit jeweils sehr andersartigen Fokussierungen. Diese mögen partiell „zu wenig differenziert“ sein, sind aber auch eher als Teil eines noch fortdauernden Prozesses der Auseinandersetzung mit der Stasi-Thematik zu lesen. Tatsächlich wird man gespannt sein dürfen, ob sich das mediale Bild von der Stasi in Zukunft eher weiter ausdifferenzieren oder wieder verengen wird.

Funding Open Access funding provided by Projekt DEAL.

Open Access Dieser Artikel wird unter der Creative Commons Namensnennung 4.0 International Lizenz veröffentlicht, welche die Nutzung, Vervielfältigung, Bearbeitung, Verbreitung und Wiedergabe in jeglichem Medium und Format erlaubt, sofern Sie den/die ursprünglichen Autor(en) und die Quelle ordnungsgemäß nennen, einen Link zur Creative Commons Lizenz beifügen und angeben, ob Änderungen vorgenommen wurden.

Die in diesem Artikel enthaltenen Bilder und sonstiges Drittmaterial unterliegen ebenfalls der genannten Creative Commons Lizenz, sofern sich aus der Abbildungslegende nichts anderes ergibt. Sofern das betreffende Material nicht unter der genannten Creative Commons Lizenz steht und die betreffende Handlung nicht nach gesetzlichen Vorschriften erlaubt ist, ist für die oben aufgeführten Weiterverwendungen des Materials die Einwilligung des jeweiligen Rechteinhabers einzuholen.

Weitere Details zur Lizenz entnehmen Sie bitte der Lizenzinformation auf http://creativecommons.org/ licenses/by/4.0/deed.de. 